\title{
Properties of the third harmonic of the radiation from self-amplified spontaneous emission free electron laser
}

\author{
E. L. Saldin, E. A. Schneidmiller, and M. V. Yurkov \\ Deutsches Elektronen-Synchrotron (DESY), Hamburg, Germany
}

(Received 10 January 2006; published 23 March 2006)

\begin{abstract}
Recent theoretical and experimental studies have shown that the self-amplified spontaneous emission free-electron laser (SASE FEL) with a planar undulator holds a potential for generation of relatively strong coherent radiation at the third harmonic of the fundamental frequency. Here we present a detailed study of the nonlinear harmonic generation in the SASE FEL obtained with a time-dependent FEL simulation code. Using similarity techniques we present universal dependencies for temporal, spectral, and statistical properties of the odd harmonics of the radiation from SASE FEL. In particular, we derived universal formulas for radiation power of the odd harmonics at saturation. It was also found that coherence time at saturation falls inversely proportional to the harmonic number, and relative spectrum bandwidth remains constant with the harmonic number.
\end{abstract}

DOI: 10.1103/PhysRevSTAB.9.030702

PACS numbers: 41.60.Cr, 41.50.+h, 42.55.Vc

\section{INTRODUCTION}

Radiation of the electron beam in the planar undulator contains rich harmonic spectrum. This refers to both incoherent and coherent radiation as well. In recent years a significant effort of researchers has been devoted to studying the process of higher harmonic generation in high-gain free-electron lasers [1-11]. Such an interest has been mainly driven by practical needs for prediction of the properties of x-ray free-electron lasers. A fraction of a higher harmonic content is very important for users planning experiments at the x-ray FEL facility. On the one hand, higher harmonics constitute a rather harmful background for some classes of experiments. On the other hand, higher harmonic radiation can significantly extend the operating band of the user facility. In both cases it is highly desirable to know properties of the higher harmonic radiation. Analytical techniques have been used to predict properties of the higher harmonics for the FEL amplifier operating in the linear mode $[8,9]$. However, the greatest fraction of radiation power is produced in the nonlinear regime, and a set of assumptions needs to be accepted in order to estimate saturation power of higher harmonics on the base of extrapolation of analytical results. As for statistical properties, they could not be extrapolated from linear theory at all. Many studies have been performed with numerical simulation codes. These studies developed in two directions. The first direction is investigations of higher harmonic phenomena by means of steady-state codes [4-7]. Even though the results of these studies are applicable to externally seeded FEL amplifiers, it is relevant to appreciate that they gave the first predictions for high radiation power in higher harmonics of SASE FEL. Another direction was an extraction of time structure for beam bunching from the time-dependent simulation code with the subsequent use of analytical formulas of the linear theory [8]. Giving an estimate for power, such an approach does not allow the describing of statistical properties of output radiation.

In this paper we perform a comprehensive study of the statistical properties of the odd harmonic radiation from SASE FEL. The study is performed in the framework of a one-dimensional model with time-dependent simulation code FAST $[12,13]$ upgraded for the simulation of higher harmonic generation. We restrict our study with odd harmonics produced in the SASE FEL. We omit from consideration an effect of self-consistent amplification of higher harmonics. In other words, we solve only the electrodynamic problem assuming that particle motion is governed by the fundamental harmonic. The latter approximation is valid when power in higher harmonics is much less than in the fundamental. This does not limit practical applicability of the results: it has been shown in earlier papers that the growth rate of higher harmonics is too small to produce a visible increase of the coherent amplification above shot noise in x-ray FELs [8]. Under this approximation and using similarity techniques we derive universal relations describing general properties of the odd harmonics in the SASE FEL: power, statistical and spectral properties. The results are illustrated for the 3rd and 5th harmonic having practical importance for $\mathrm{x}$-ray FELs.

\section{BASIC RELATIONS}

The magnetic field of the planar undulator is of the form

$$
H_{z}(z)=H_{\mathrm{w}} \cos \left(2 \pi z / \lambda_{\mathrm{w}}\right) \text {, }
$$

where $\lambda_{\mathrm{w}}$ is the undulator period and $H_{\mathrm{w}}$ is the peak magnetic field. In the SASE FEL the radiation is produced by the electron beam during a single pass of the undulator [14-17]. The amplification process starts from shot noise in the electron beam. During the amplification process a powerful, coherent radiation is produced having narrow band near resonance wavelength: 


$$
\lambda_{0}=\frac{\lambda_{\mathrm{w}}}{2 \gamma^{2}}\left(1+K^{2}\right)
$$

where $K=e \lambda_{\mathrm{w}} H_{\mathrm{w}} /\left(2 \sqrt{2} \pi m c^{2}\right)$ is the rms undulator parameter, $\gamma$ is the relativistic factor, and $(-e)$ and $m$ are the charge and mass of electron, respectively.

In the framework of the one-dimensional model we consider the amplification of the plane electromagnetic wave by the electron beam in the undulator. When space charge and energy spread effects can be neglected, operation of an FEL amplifier is described in terms of the gain parameter $\Gamma$ and efficiency parameter $\rho$ (see, e.g., $[13,16]$ ):

$$
\Gamma=\left[\frac{\pi j_{0} K_{1}^{2}}{I_{A} \lambda_{\mathrm{w}} \gamma^{3}}\right]^{1 / 3}, \quad \rho=\frac{\lambda_{\mathrm{w}} \Gamma}{4 \pi} .
$$

Here $j_{0}$ is the beam current density $I_{A}=m c^{3} / e \simeq 17 \mathrm{kA}$ and $\omega=2 \pi c / \lambda$ is the frequency of the electromagnetic wave. The coupling factor $K_{h}$ is given by

$$
K_{h}=K(-1)^{(h-1) / 2}\left[J_{(h-1) / 2}(Q)-J_{(h+1) / 2}(Q)\right],
$$

where $Q=h K^{2} /\left[2\left(1+K^{2}\right)\right]$. The FEL amplifier is a resonance device with an amplification bandwidth about $\Delta \omega / \omega_{0} \simeq 2 \rho$ around resonance frequency $\omega_{0}=$ $2 \pi c / \lambda_{0}$. In the linear stage of amplification the radiation power $W$ grows exponentially along the undulator length, $W \propto \exp \left[2 z / L_{\mathrm{g}}\right]$, and the field gain length is about $L_{\mathrm{g}} \simeq$ $2 /(\Gamma \sqrt{3})$. Saturation of the FEL amplifier occurs when relative energy loss by the electrons at one field gain length becomes about saturation parameter $\rho$.

A complete description of the startup from shot noise in the FEL amplifier can be performed only with timedependent simulations of the FEL process. We do not present here general technical details of the timedependent simulations; they have been described previously in detail $[12,13]$. The only add-on is the particle loading tool, but it is similar to that described in other papers (see, e.g., [18] and references therein). We note only that under accepted approximation (the particle's dynamics is governed by the fundamental harmonic) we can simply calculate odd harmonics from particle distribution, and amplitude of the electric field scales as

$$
E(z, t) \propto K_{h} \int_{0}^{z} a_{h}\left(z^{\prime}, t-z^{\prime} / c\right) d z^{\prime}
$$

where $a_{h}$ is $h$ th harmonic of the beam bunching. Thus, we find that the coupling factor $K_{h}$, and time-dependent integral of the beam bunching become factorized. This allows us to extract the universal ratio of the power of higher harmonics to the power of fundamental harmonic.

\section{STATISTICAL PROPERTIES OF THE ODD HARMONICS OF THE RADIATION FROM SASE FEL}

In this section we present the results of numerical studies of the operation of the SASE FEL in the linear and nonlinear regime. In the framework of the accepted model, the input parameter of the system is the number of cooperating electrons $N_{\mathrm{c}}=I /\left(e \rho \omega_{0}\right)$, where $I$ is beam current. Most of the statistical characteristics of the SASE FEL process are functions of $N_{\mathrm{c}}$ only at fixed $z$ coordinate $[13,19]$. A typical range of the values of $N_{\mathrm{c}}$ is $10^{6}-10^{9}$ for the SASE FELs of wavelength range from $x$-ray to infrared. The numerical results, presented in this section, are calculated for the value $N_{\mathrm{c}}=3 \times 10^{7}$ which is typical for a VUV FEL. It is worth mentioning that the dependence of the output parameters of the SASE FEL on the value of $N_{\mathrm{c}}$ is rather weak, in fact logarithmic. Therefore, the obtained results are pretty general and can be used for the estimation of the parameters of actual devices with sufficient accuracy.

\section{A. Temporal characteristics}

Figure 1 presents a typical time structure of the 1st and 3 rd harmonic of the radiation from a SASE FEL at different undulator length $\hat{z}=\Gamma z=10-13$. Normalized power of $h$ th harmonic is defined as $\hat{\eta}_{h}=\left\langle W_{h}\right\rangle \times$ $\left(K_{1} / K_{h}\right)^{2} /\left(\rho W_{\mathrm{b}}\right)$. Here $\left\langle W_{h}\right\rangle$ is averaged radiation power in the $h$ th harmonic, and $W_{\mathrm{b}}=\gamma m c^{2} I / e$ is electron beam power. The longitudinal coordinate along the pulse is $\hat{s}=$ $\rho \omega_{0}\left(z / \bar{v}_{z}-t\right)$. Here $\bar{v}_{z}=v-c K^{2} /\left(2 \gamma^{2}\right)$ is the velocity of the electron along the $z$ axis averaged over the undulator period. The head of the pulse is located in the positive direction of $\hat{s}$. The plot for the averaged power of the 1st harmonic is shown in Fig. 2 with a solid line. It is seen that saturation is achieved at the undulator $\hat{z}=13$. Saturation length is well described in terms of the number of cooperating electrons $N_{\mathrm{c}}[13,19]$ :

$$
\hat{z}_{\mathrm{sat}} \simeq 3+\frac{1}{\sqrt{3}} \ln N_{\mathrm{c}} .
$$

The normalized efficiency at saturation, $\hat{\eta}_{\text {sat }}=$ $\left\langle W_{\text {sat }}\right\rangle /\left(\rho W_{\mathrm{b}}\right) \simeq 1.08$, is almost independent of the value of $N_{\mathrm{c}}$. Dashed and dotted lines show a normalized power ratio, $\hat{\eta}_{h} / \hat{\eta}_{1}=\left(\left\langle W_{h}\right\rangle /\left\langle W_{1}\right\rangle\right) \times\left(K_{1} / K_{h}\right)^{2}$, for the 3rd and the 5th harmonic. Universal functions for the ratio $\left(K_{h} / K_{1}\right)^{2}$ are plotted in Fig. 3. One can notice that power of the higher harmonics becomes above the shot noise level only in the end of the linear regime. This becomes clear if one takes into account that the shot noise level of the beam bunching is about $1 / \sqrt{N_{\mathrm{c}}}$. We consider an example typical for VUV FEL with $N_{\mathrm{c}}=3 \times 10^{7}$ which corresponds to the shot noise beam bunching $a \simeq 2 \times 10^{-4}$. When the FEL amplifier operates in the linear regime, odd harmonics grow as $a_{1}^{h}$, and we expect from this simple physical 

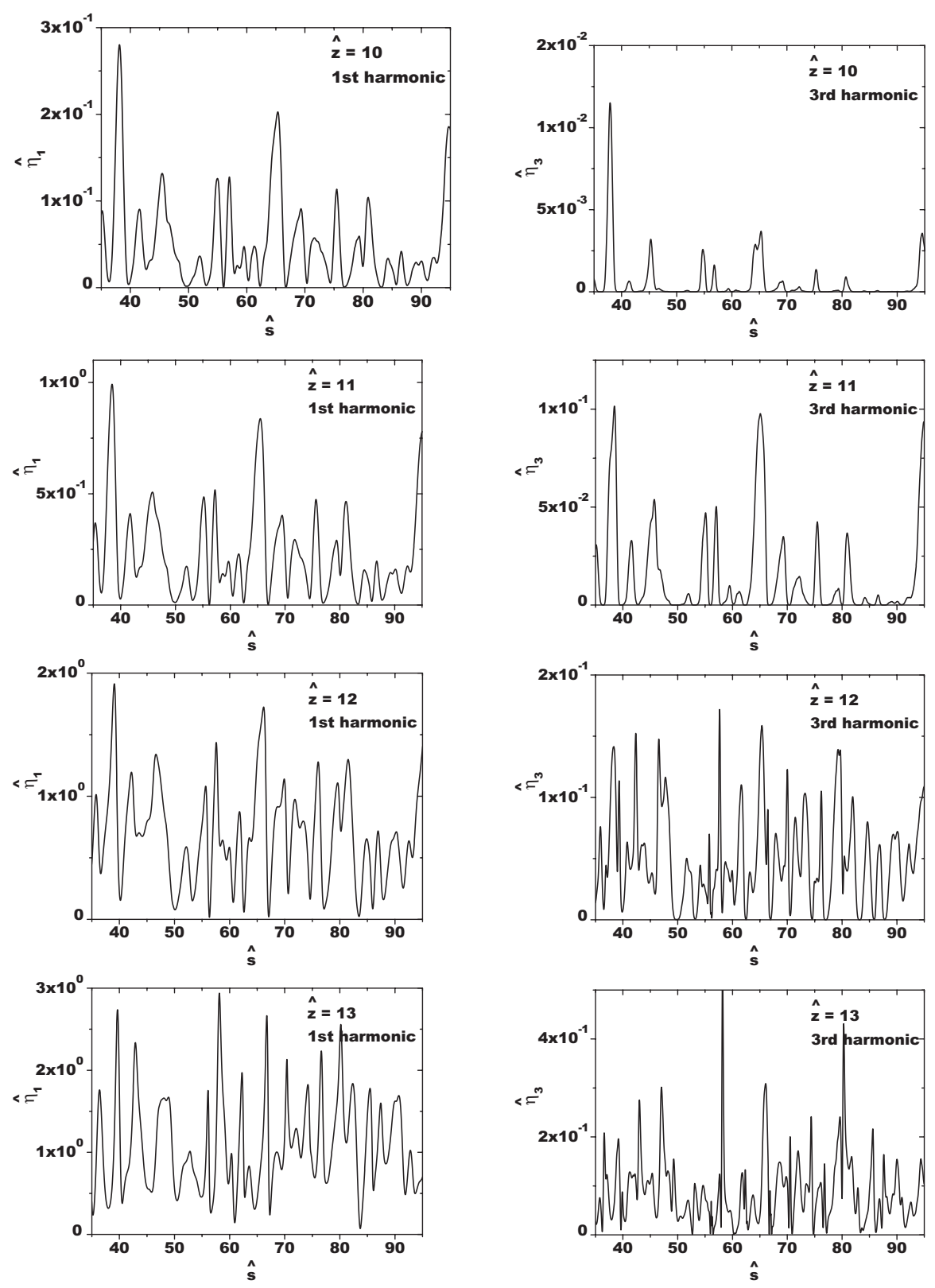

FIG. 1. Normalized power in the radiation pulse versus $\hat{s}=\rho \omega_{0}\left(z / \bar{v}_{z}-t\right)$ at different lengths of the FEL amplifier $\hat{z}=10-13$. Left and right columns correspond to the fundamental and 3rd harmonic, respectively.

estimation that coherent contribution into higher harmonics can exceed the shot noise level only for the values of the beam bunching at the fundamental harmonic $a_{1} \gtrsim 0.1$, i.e., in the end of the linear regime. Note that shot noise level becomes higher when approaching the $\mathrm{x}$-ray region.

The plots presented in Fig. 1 allow one to trace the evolution of the 3rd harmonic power from $\hat{z}=10$ (when it just started to exceed shot noise level) up to saturation point $\hat{z}=13$. At $\hat{z}=10-11$ SASE FEL operates in the high-gain linear regime, and the beam bunching in the fundamental harmonic is small, $\left|a_{1}\right| \ll 1$. In this case one can expect a well-known mechanism of the higher harmonic generation, i.e. $a_{h} \propto a_{1}^{h}$, and spikes of the $3 \mathrm{rd}$ harmonic radiation become rather pronounced. However, the noise nature of the SASE FEL makes a big difference in the behavior of growth rates with respect to predictions given in the framework of steady-state simulations [4,5]. Analyzing the plot for the power growth rate (see Fig. 4) we can state that in practical situation the prediction of the steady-state theory (the growth rate of higher harmonics is proportional to the harmonic number) is valid only for the 3rd harmonic, but only on a short piece of undulator close to saturation, of about one gain length. Also, a prediction for the relation between averaged values of the beam bunching at the third harmonic, $\left\langle\left|a_{3}\right|^{2}\right\rangle=6\left\langle\left|a_{1}\right|^{2}\right\rangle^{3}$, holds only approximately, and is strongly violated for higher harmonics, because of a strong contribution of the shot noise. This feature of the SASE FEL has been highlighted 


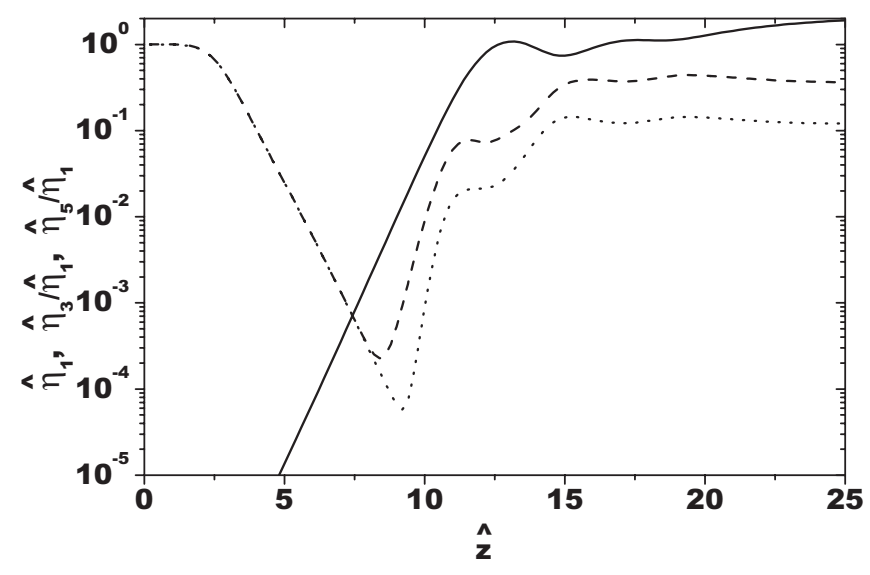

FIG. 2. Normalized averaged power of a fundamental harmonic of SASE FEL, $\hat{\eta}_{1}=P_{1} /\left(\rho P_{\text {beam }}\right)$, as a function of a normalized undulator length (solid line). Dashed and dotted lines show a normalized power ratio $\hat{\eta}_{h} / \hat{\eta}_{1}=\left(W_{h} / W_{1}\right) \times\left(K_{1} / K_{h}\right)^{2}$ for the 3rd and 5th harmonic.

qualitatively in early papers [8] with the analysis of simulation results obtained with code GINGER [3]. Here we just presented a more quantitative study.

The plots in Fig. 2 present a general result for a ratio of the power in the higher harmonics with respect to the fundamental one. For the saturation we find a universal dependency:

$$
\left.\frac{\left\langle W_{3}\right\rangle}{\left\langle W_{1}\right\rangle}\right|_{\text {sat }}=0.094 \times \frac{K_{3}^{2}}{K_{1}^{2}},\left.\quad \frac{\left\langle W_{5}\right\rangle}{\left\langle W_{1}\right\rangle}\right|_{\text {sat }}=0.03 \times \frac{K_{5}^{2}}{K_{1}^{2}} .
$$

Asymptotic values for a large value of the undulator parameter are $\left(K_{3} / K_{1}\right)^{2} \simeq 0.22$ and $\left(K_{5} / K_{1}\right)^{2} \simeq 0.11$. Thus, we can state that contribution of the 3rd harmonic into the total radiation power of SASE FEL at saturation could not exceed a level of $2 \%$. Thus, its influence on the beam

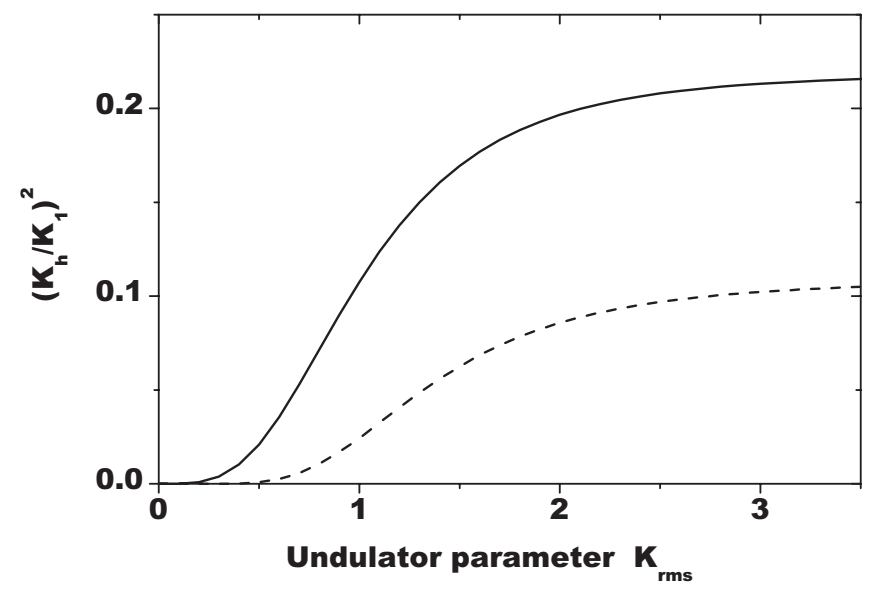

FIG. 3. Ratio of coupling factors, $\left(K_{h} / K_{1}\right)^{2}$, for the 3rd (solid line) and the 5 th (dashed line) harmonics with respect to the fundamental harmonic versus rms value of undulator parameter $K_{\text {rms. }}$.

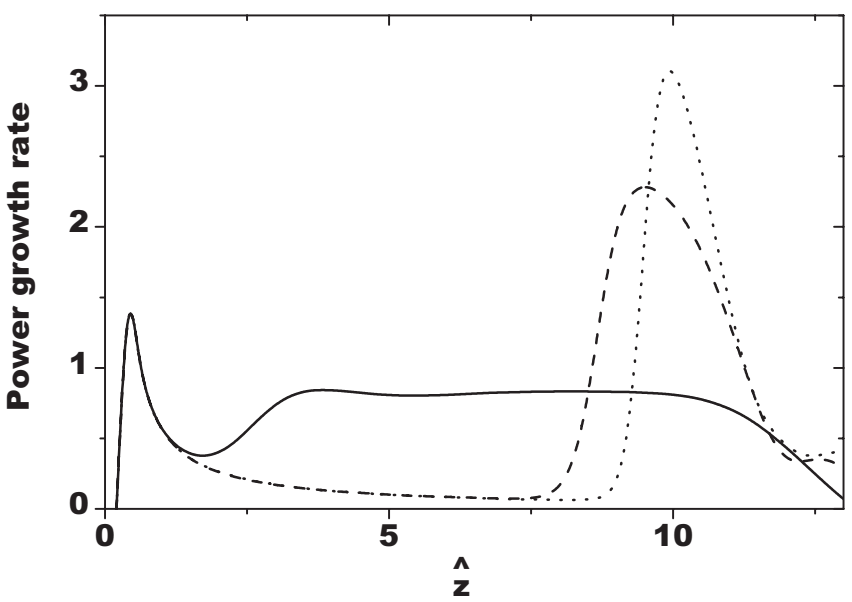

FIG. 4. Normalized power growth rate for the 1st, 3rd, and 5th harmonic (solid, dashed, and dotted line, respectively).

dynamics should be small. This result justifies a basic assumption used for the derivation of a universal relation (6). A contribution of the 5th harmonic into the total power at saturation could not exceed the value of $0.3 \%$.

Another important topic is the impact of electron beam quality on the nonlinear harmonic generation process. In the framework of the one-dimensional theory this effect is described with the energy spread parameter $\hat{\Lambda}_{\mathrm{T}}^{2}[13]$ :

$$
\hat{\Lambda}_{\mathrm{T}}^{2}=\frac{\left\langle(\Delta E)^{2}\right\rangle}{\rho^{2} E_{0}^{2}}
$$

where $\left\langle(\Delta E)^{2}\right\rangle$ is the rms energy spread, and $E_{0}=\gamma m c^{2}$ is nominal energy of electrons. Thus, the result given by (6) is generalized to the case of finite energy spread with the plot presented in Fig. 5. We see that the energy spread in the electron beam suppresses power of the higher harmonics. Within the practical range of $\hat{\Lambda}_{\mathrm{T}}^{2}$ this suppression can be

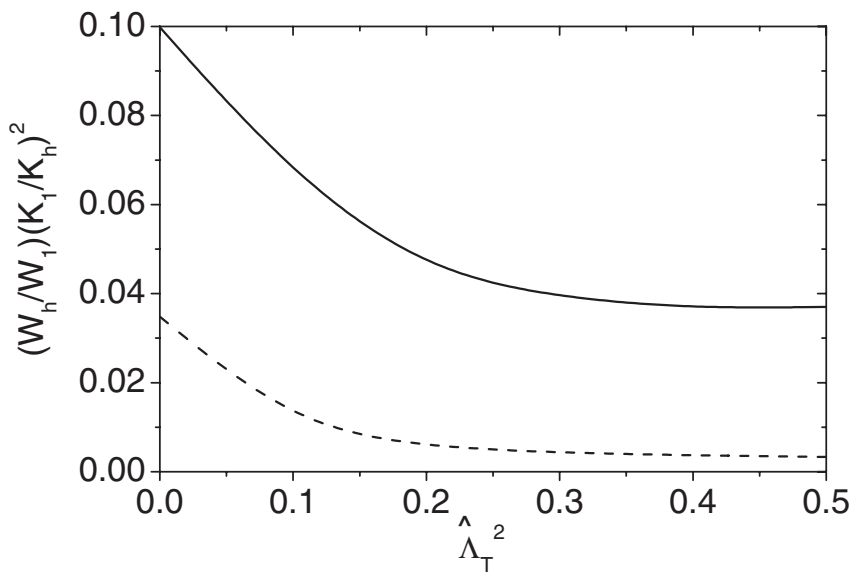

FIG. 5. Normalized power ratio at saturation $\left(W_{h} / W_{1}\right) \times$ $\left(K_{1} / K_{h}\right)^{2}$ for the 3 rd (solid line) and 5 th (dashed line) harmonic as a function of energy spread parameter $\hat{\Lambda}_{\mathrm{T}}^{2}$. SASE FEL opeartes at saturation. 
about a factor of 3 for the 3rd harmonic, and about an order of magnitude for the 5th harmonic. For practical estimations one should use an effective value of the energy spread describing the contribution of the energy spread and the emittance to the longitudinal velocity spread [13]:

$$
\frac{\left\langle(\Delta E)^{2}\right\rangle_{\mathrm{eff}}}{E_{0}^{2}}=\frac{\left\langle(\Delta E)^{2}\right\rangle}{E_{0}^{2}}+\frac{2 \gamma_{z}^{4} \epsilon^{2}}{\beta^{2}},
$$

where $\gamma_{z}$ is the longitudinal relativistic factor $\left[\gamma_{z}^{2}=\right.$ $\left.\gamma^{2} /\left(1+K^{2}\right)\right], \epsilon$ is beam emittance, and $\beta$ is the focusing beta function. The plot in Fig. 5 covers a practical range of parameters for x-ray FELs. The saturation length at $\hat{\Lambda}_{\mathrm{T}}^{2}=$ 0.5 is increased by a factor of 1.5 with respect to the "cold" beam case $\hat{\Lambda}_{\mathrm{T}}^{2}=0$.

\section{B. Probability distributions}

The next step of our study is the behavior of the probability distribution of the instantaneous power. In Fig. 6 we show the normalized rms deviation of the instantaneous radiation power $\sigma_{\mathrm{w}}=\left\langle(W-\langle W\rangle)^{2}\right\rangle^{1 / 2} /\langle W\rangle$ as a function of the undulator length. We see that at the initial stage of SASE FEL operation rms deviation of the instantaneous power is equal to one for all harmonics. This is a consequence of startup from the shot noise in the electron beam: statistical properties of the undulator radiation and of the radiation from SASE FEL operating in the linear regime are governed by Gaussian statistics $[13,19]$. One of the important features of Gaussian statistics is that the normalized rms deviation of instantaneous radiation power is equal to unity. For the fundamental harmonic statistics of the radiation becomes non-Gaussian when the amplification process enters the nonlinear mode [13,19]. For the higher harmonics non-Gaussian statistics takes place when the nonlinear harmonic generation starts to dominate above

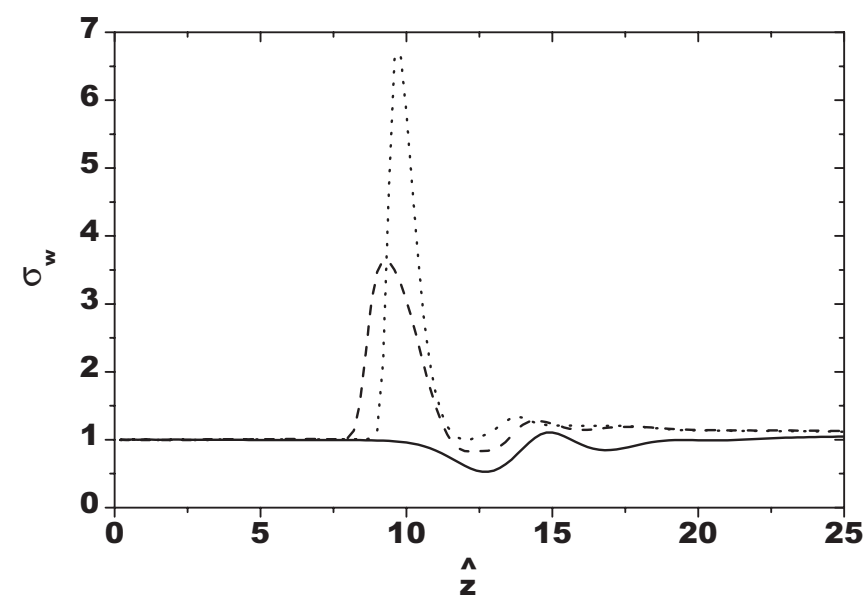

FIG. 6. Normalized rms deviation of the fluctuations of the instantaneous radiation power as a function of the normalized undulator length. Solid, dashed, and dotted lines correspond to the fundamental, 3rd, and 5th harmonic, respectively. incoherent radiation (at $\hat{z} \geq 8$ in the present numerical example). Analytical theory of nonlinear harmonic generation [8] predicts the value of $\sigma_{\mathrm{w}} \simeq 4$ for the third harmonic. Analysis of the relevant curve in Fig. 6 shows that this prediction holds approximately in a short piece of the undulator length only. As we explained above, this is due to the fact that nonlinear harmonic generation starts to dominate above incoherent radiation only at the values of the beam bunching at the fundamental harmonic $a_{1} \sim 0.1$. However, at such a value of the beam bunching the amplification process is visibly affected by nonlinear effects.

Probability density distributions for the instantaneous power of the fundamental and the 3rd harmonic are presented in Fig. 7. SASE radiation is a stochastic object and at a given time it is impossible to predict the amount of energy which flows to a detector. The initial modulation of the electron beam is defined by the shot noise and has a white spectrum. The high-gain FEL amplifier cuts and amplifies only a narrow frequency band of the initial spectrum $\Delta \omega / \omega \ll 1$. In the time domain, the temporal structure of the fundamental harmonic radiation is chaotic with many random spikes, with a typical duration given by the inverse width of the spectrum envelope. Even without performing numerical simulations, we can describe some general properties of the fundamental harmonic of the radiation from the SASE FEL operating in the linear regime. Indeed, in this case we deal with Gaussian statistics. As a result, the probability distribution of the instantaneous radiation intensity $W$ should be the negative exponential probability density distribution $[13,19]$ :

$$
p(W)=\frac{1}{\langle W\rangle} \exp \left(-\frac{W}{\langle W\rangle}\right)
$$

Here one should realize clearly that the notion of instantaneous intensity refers to a certain moment in time, and that the analysis must be performed over an ensemble of pulses. Also, the energy in the radiation pulse $E_{\text {rad }}$ should fluctuate in accordance with the gamma distribution $[13,19]$ :

$$
p\left(E_{\mathrm{rad}}\right)=\frac{M^{M}}{\Gamma(M)}\left(\frac{E_{\mathrm{rad}}}{\left\langle E_{\mathrm{rad}}\right\rangle}\right)^{M-1} \frac{1}{\left\langle E_{\mathrm{rad}}\right\rangle} \exp \left(-M \frac{E_{\mathrm{rad}}}{\left\langle E_{\mathrm{rad}}\right\rangle}\right),
$$

where $\Gamma(M)$ is the gamma function of argument $M=$ $1 / \sigma_{\text {rad }}^{2}$, and $\sigma_{\text {rad }}^{2}=\left\langle\left(E_{\text {rad }}-\left\langle E_{\text {rad }}\right\rangle\right)^{2}\right\rangle /\left\langle E_{\text {rad }}\right\rangle^{2}$ is the normalized dispersion of the energy distribution. These properties are well known in statistical optics as properties of completely chaotic polarized radiation [20].

The statistics of the high-harmonic radiation from the SASE FEL changes significantly with respect to the fundamental harmonic (e.g., with respect to Gaussian statistics). It is interesting in our case to be able to determine the probability density function of instantaneous intensity of SASE radiation after it has been subjected to nonlinear transformation. We know the probability density function 

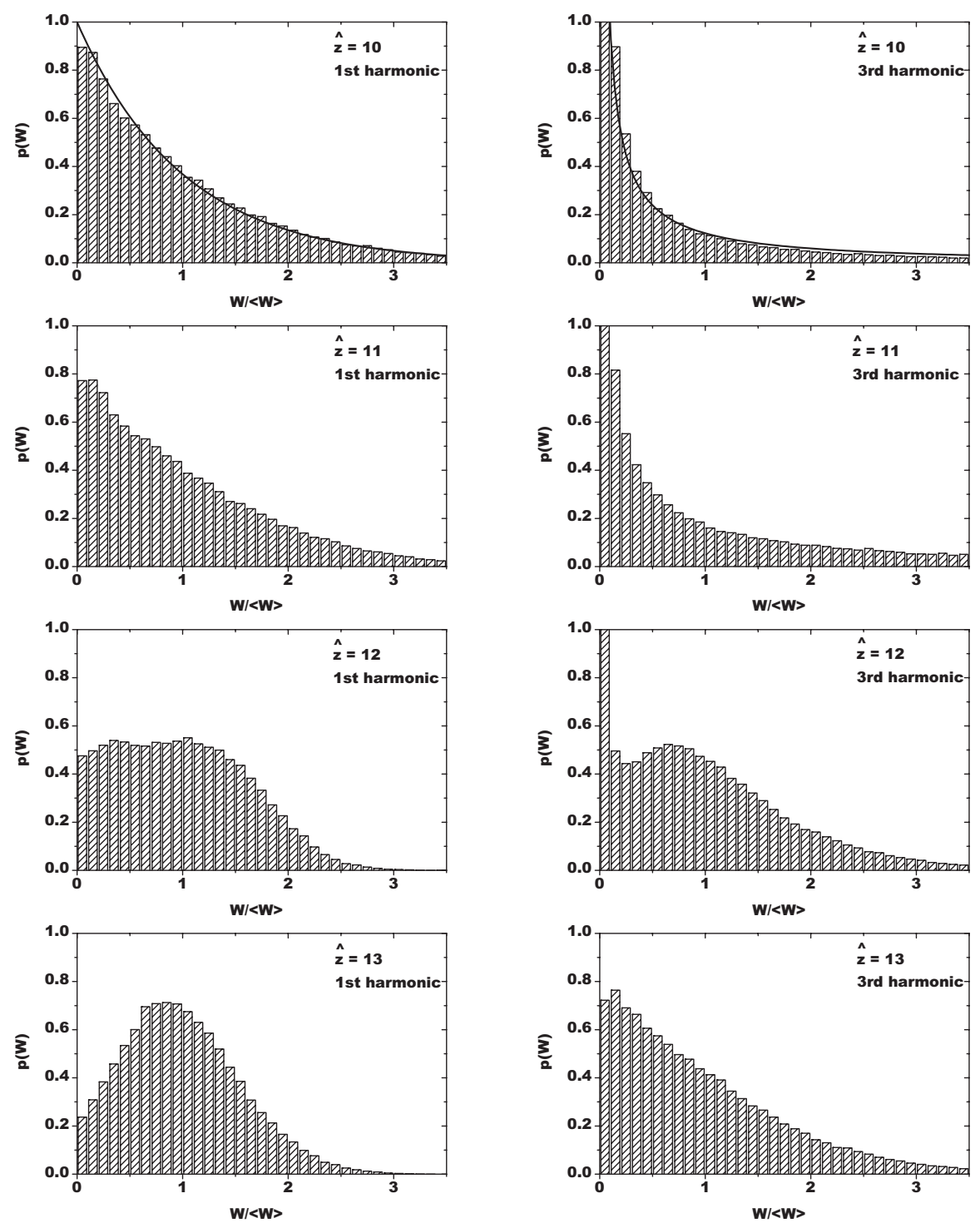

FIG. 7. Probability distribution of instantaneous radiation power at different lengths of the FEL amplifier $\hat{z}=10-13$. Left and right columns correspond to the fundamental and 3rd harmonic, respectively. Solid line shows probability density function (9).

$p(W)=\langle W\rangle^{-1} \exp (-W /\langle W\rangle)$ of the fundamental intensity $W$, and $W$ is subjected to a transformation $z=(W)^{h}$. The problem is then to find the probability density function $p(z)$. It can be readily shown that this probability distribution is [21]:

$$
p(z)=\frac{z}{h\langle W\rangle} z^{(1-h) / h} \exp \left(-z^{1 / h} /\langle W\rangle\right)
$$

Using this distribution we get the expression for the mean value: $\langle z\rangle=h !\langle W\rangle^{h}$. Thus, the $h$ th-harmonic radiation for the SASE FEL has an intensity level roughly $h$ ! times larger than the corresponding steady-state case, but with more shot-to-shot fluctuations compared to the fundamental [8]. Nontrivial behavior of the intensity of the high harmonic reflects the complicated nonlinear trans- formation of the fundamental harmonic statistics. One can see that Gaussian statistics is no longer valid. Upper plots in Fig. 7 give an illustration to these consideration. Even though in practical example we do not have a pure linear amplification regime, the probability density functions for instantaneous power follow prediction rather well (9).

Analysis of the probability distributions in Fig. 7 shows that in the nonlinear regime, near the saturation point, the distributions change significantly with respect to the linear regime for both the fundamental and the 3rd harmonic. An important message is that at the saturation point, the 3rd harmonic radiation exhibits a much more noisy behavior (nearly negative exponential) while stabilization of the fluctuations of the fundamental harmonics takes place. 


\section{Correlation functions}

The first- and second-order time correlation functions are defined as follows:

$$
\begin{aligned}
& g_{1}\left(t-t^{\prime}\right)=\frac{\left\langle\tilde{E}(t) \tilde{E}^{*}\left(t^{\prime}\right)\right\rangle}{\left[\left\langle|\tilde{E}(t)|^{2}\right\rangle\left\langle\left|\tilde{E}\left(t^{\prime}\right)\right|^{2}\right\rangle\right]^{1 / 2}}, \\
& g_{2}\left(t-t^{\prime}\right)=\frac{\left\langle|\tilde{E}(t)|^{2}\left|\tilde{E}\left(t^{\prime}\right)\right|^{2}\right\rangle}{\left\langle|\tilde{E}(t)|^{2}\right\rangle\left\langle\left|\tilde{E}\left(t^{\prime}\right)\right|^{2}\right\rangle} .
\end{aligned}
$$

In Fig. 8 we show the evolution of the time correlation functions of first and second order. At each normalized position along the undulator, $\hat{z}$, they are plotted versus the normalized variable $\hat{\tau}=\rho \omega_{0}\left(t-t^{\prime}\right)$. The upper plot in Fig. 8 corresponds to the linear stage of SASE FEL operation. In the case of the fundamental harmonic we deal with a Gaussian random process and the relation between the correlation functions holds for $g_{2}\left(t-t^{\prime}\right)=1+\mid g_{1}(t-$ $\left.t^{\prime}\right)\left.\right|^{2}$. This feature does not hold place for higher harmonics. The nontrivial behavior of the correlation functions reflects the complicated nonlinear evolution of the SASE FEL process. The second-order correlation function of zero argument, $g_{2}(0)$, takes values smaller or larger than 2 , but always larger than unity. Note that there is a simple relation between $g_{2}(0)$ and the normalized rms power deviation: $g_{2}(0)=1+\sigma_{\mathrm{w}}^{2}$ (see Fig. 6). It is a well-known result of statistical optics that the cases of $g_{2}(0)=1$ and $g_{2}(0)=2$ correspond to stabilized single-mode laser radiation and to completely chaotic radiation from a thermal source, respectively. The values of $g_{2}(0)$ between 1 and 2 belong to some intermediate situation. In classical optics, a radiation source with $g_{2}(0)<1$ cannot exist but the case of
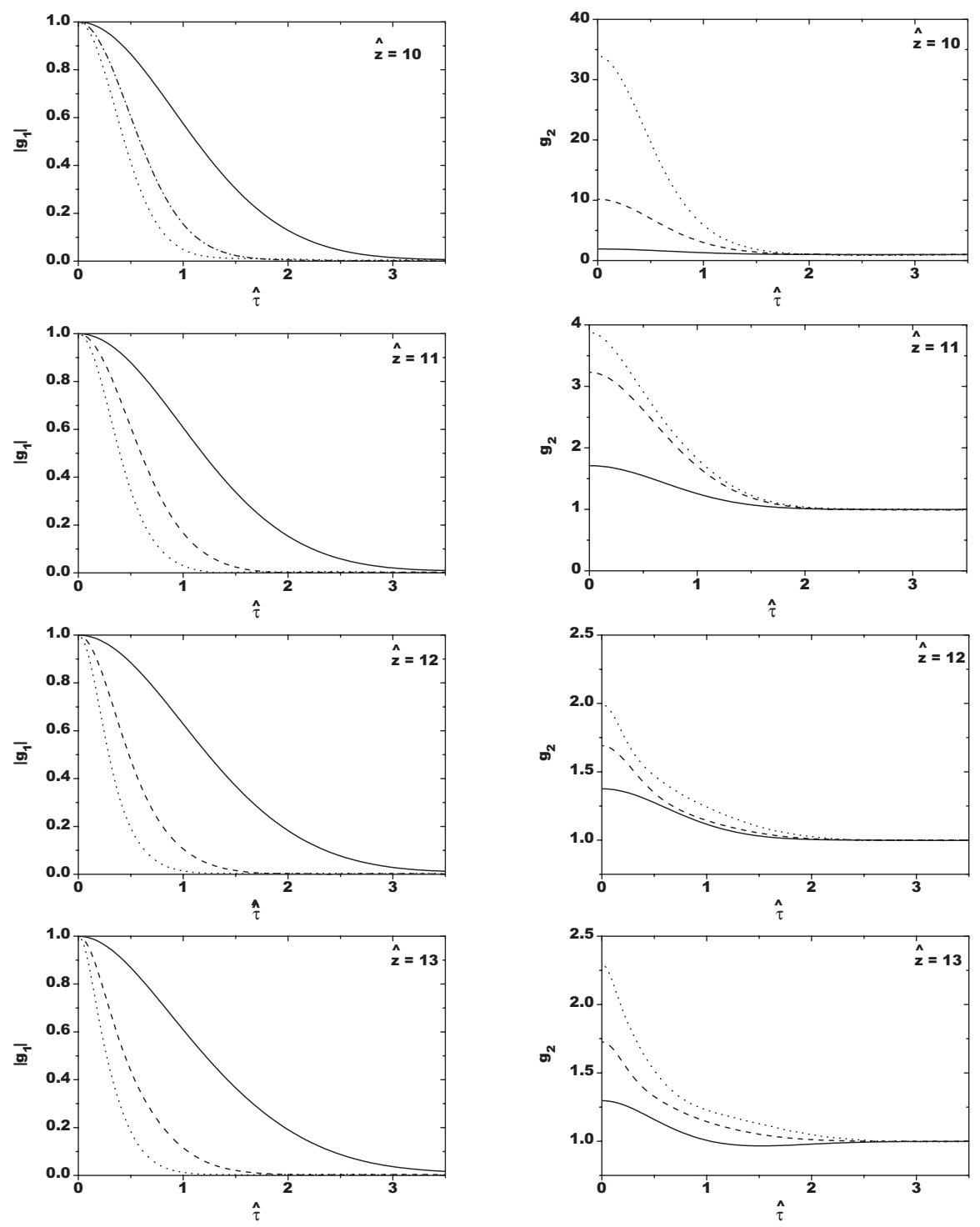

FIG. 8. First (left column) and second (right column) order correlation function at different lengths of the FEL amplifier $\hat{z}=10-13$. Solid, dashed, and dotted lines correspond to the fundamental, 3rd, and 5th harmonic, respectively 
$g_{2}(0)>2$ is possible. As one can see from Fig. 8, the latter phenomenon (known as superbunching) occurs for higher harmonics of SASE FEL, or for the fundamental one when the SASE FEL is operating in the nonlinear regime.

In Fig. 9 we present the dependence on the undulator length of the normalized coherence time $\hat{\tau}_{\mathrm{c}}=\rho \omega_{0} \tau_{\mathrm{c}}$, where $\tau_{\mathrm{c}}$ is

$$
\tau_{\mathrm{c}}=\int_{-\infty}^{\infty}\left|g_{1}(\tau)\right|^{2} d \tau
$$

For the fundamental harmonic the coherence time achieves its maximal value near the saturation point and then decreases drastically. The maximal value of $\hat{\tau}_{\mathrm{c}}$ depends on the saturation length and, therefore, on the value of the parameter $N_{\mathrm{c}}$. With logarithmic accuracy we have the following expression for the coherence time of the fundamental harmonic:

$$
\left(\hat{\tau}_{\mathrm{c}}\right)_{\max } \simeq \sqrt{\frac{\pi \ln N_{\mathrm{c}}}{18}} .
$$

The longitudinal coherence for higher harmonics evolves in three different stages. Initially (up to $\hat{z}=7-8$, see Fig. 9) coherence time increases linearly as it should be for the spontaneous emission of the radiation from the undulator. When the process of nonlinear harmonic generation starts to dominate above spontaneous emission, coherence time drops sharply. At the positions around $\hat{z}=$ 10-11 we obtain some plateau where the ratio of the coherence time of the $h$ th harmonic to that of the 1 st harmonic scales as $1 / \sqrt{h}$. At these distances SASE FEL still operates in the exponential regime when amplitude of the beam bunching is visibly less than unity, and the intensity of the $h$ th-harmonic scales as $I_{1}^{h}$. Such a mechanism of nonlinear harmonic generation leads to the scaling of the coherence time as $1 / \sqrt{h}$. To explain this we refer to

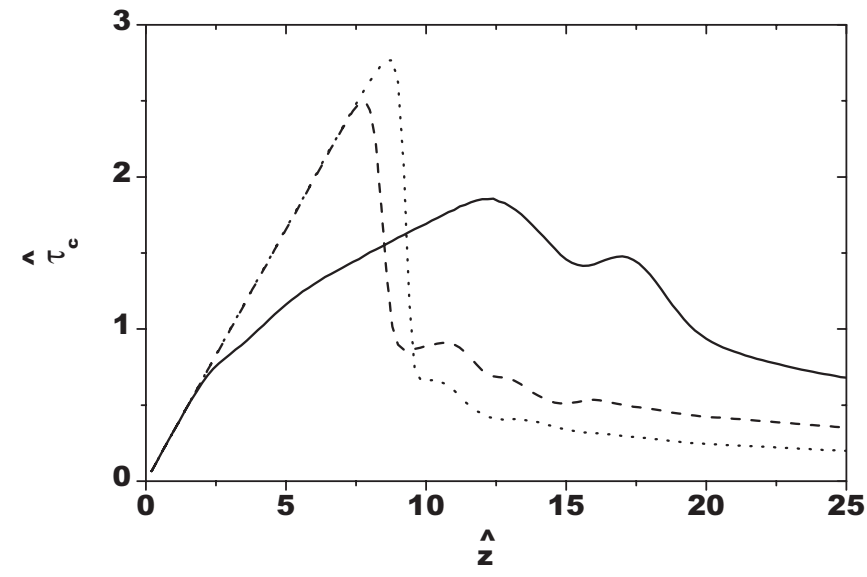

FIG. 9. Normalized coherence time of a SASE FEL as a function of normalized undulator length. Solid, dashed, and dotted lines correspond to the fundamental, 3rd, and 5th harmonic, respectively.
Fig. 1 presenting a temporal structure of the radiation pulse. The radiation pulse consists of a number of spikes (wave packets). For the sake of simplicity let us approximate a wave packet with Gaussian, $G_{1}(\hat{s})$, with an rms width $\sigma_{1}$. Nonlinear transformation of the intensity for the $h$ th harmonic gives us envelope $G_{h} \propto G_{1}^{h}$. Therefore, a relevant spike for the $h$ th harmonic is $\sigma_{h}=\sigma_{1} / \sqrt{h}$. In other words, sharpening of the peaks in intensity distribution leads to the suppression of coherence time for higher harmonics. When the amplification process enters the nonlinear stage $(\hat{z} \geq 11)$, the relative sharpening of the intensity peaks of higher harmonics becomes stronger, and coherence time starts to fall down again. In fact, as one can find from Fig. 8, coherence time at saturation point $(\hat{z}=13)$ for higher harmonics approximately falls inversely proportional to the harmonic number $h$.

\section{Spectral characteristics}

When comparing radiation spectra, it is convenient to use the normalized spectral density, $H(\hat{C})$, defined as

$$
\int_{-\infty}^{\infty} d \hat{C} H(\hat{C})=1
$$

Here $\hat{C}=\left[2 \pi / \lambda_{\mathrm{w}}-\omega\left(1+K^{2}\right) /\left(2 c \gamma^{2}\right)\right] / \Gamma$ is the detuning parameter. The frequency deviation, $\Delta \omega$, from the nominal value of $\omega_{h}$ can be recalculated as $\Delta \omega=$ $-2 \rho \omega_{h} \hat{C}$. Since we consider the model of a long rectangular bunch, the function $H(\hat{C})$ can be treated as the normalized spectral density of both the radiation energy and the power.

Normalized envelope of the radiation spectrum and the first order time correlation function are connected by the relation [20]:

$$
G(\Delta \omega)=\frac{1}{2 \pi} \int_{-\infty}^{\infty} d \tau g_{1}(\tau) \exp (-i \Delta \omega \tau)
$$

The temporal structures of the radiation pulses (see Fig. 1) are used for calculating the first order time correlation function (see Fig. 8). Then the radiation spectra are reconstructed by Fourier transformation of the first order time correlation function. Figure 10 shows the evolution of the radiation spectra of the SASE FEL radiation from the end of the linear regime to saturation. Note that the spectrum width of the higher harmonics from SASE FEL differs significantly from that of incoherent radiation. For the case of incoherent radiation relative spectrum width, $\Delta \omega / \omega_{h}$ scales inversely proportional to the harmonic number $h$ [22]. One can see that situation changes dramatically for the case when the nonlinear harmonic generation process starts to be dominant. At saturation we find that relative spectrum bandwidth becomes nearly the same for all odd harmonics. 

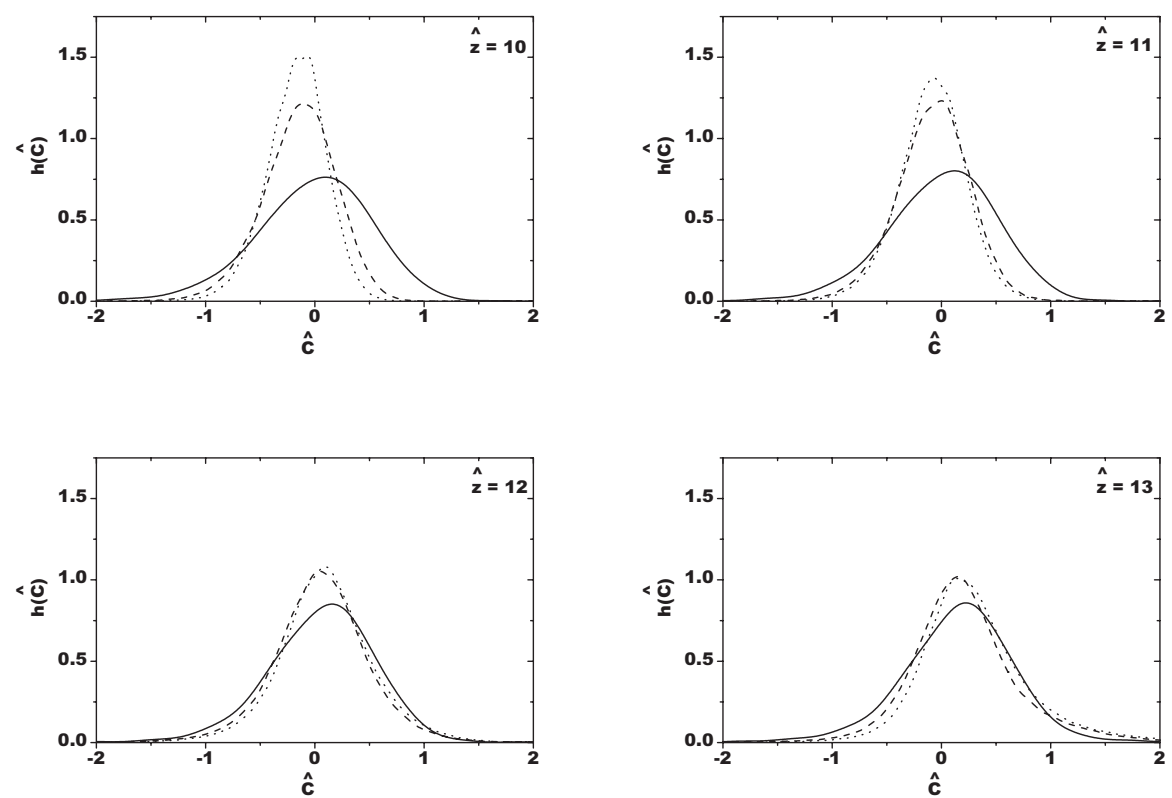

FIG. 10. Normalized spectrum at a different length of the undulator: $\hat{z}=10-13$. Solid, dashed, and dotted lines correspond to the fundamental, 3rd, and 5th harmonic, respectively.

\section{SUMMARY}

In this paper we performed a detailed study of the properties of the odd harmonic of the radiation from SASE FEL. Universal formulas for the contribution of the higher odd harmonics to the FEL power for SASE FEL operating at saturation are obtained. In the case of cold electron beam these contributions are functions of the undulator parameter $K$ only. General statistical properties of the odd harmonics of the SASE FEL operating in saturation are as follows. The power of higher harmonics is subjected to larger fluctuations than that of the fundamental one. Probability distributions of the instantaneous power of higher harmonics are close to the negative exponential distribution. The coherence time at saturation falls inversely proportional to harmonic number, and relative spectrum bandwidth remains constant with harmonic number.

[1] M. Schmitt and C. Elliot, Phys. Rev. A 34, 4843 (1986).

[2] R. Bonifacio, L. D. Salvo, and P. Pierini, Nucl. Instrum. Methods Phys. Res., Sect. A 293, 627 (1990).

[3] W. Fawley, in Proceedings of the IEEE Particle Accelerator Conference, Dallas, TX, 1995 (IEEE, Piscataway, NJ, 1995), p. 219.

[4] H. Freund, S. Biedron, and S. Milton, Nucl. Instrum. Methods Phys. Res., Sect. A 445, 53 (2000).
[5] H. Freund, S. Biedron, and S. Milton, IEEE J. Quantum Electron. 36, 275 (2000).

[6] S. Biedron et al., Nucl. Instrum. Methods Phys. Res., Sect. A 483, 94 (2002).

[7] S. Biedron et al., Phys. Rev. ST Accel. Beams 5, 030701 (2002).

[8] Z. Huang and K. J. Kim, Phys. Rev. E 62, 7295 (2000).

[9] Z. Huang and K. Kim, Nucl. Instrum. Methods Phys. Res., Sect. A 475, 112 (2001).

[10] A. Tremaine et al., Phys. Rev. Lett. 88, 204801 (2002).

[11] W. Brefeld et al., Nucl. Instrum. Methods Phys. Res., Sect. A 507, 431 (2003).

[12] E. Saldin, E. Schneidmiller, and M. Yurkov, Nucl. Instrum. Methods Phys. Res., Sect. A 429, 233 (1999).

[13] E. Saldin, E. Schneidmiller, and M. Yurkov, The Physics of Free Electron Lasers (Springer-Verlag, Berlin, 1999).

[14] A. Kondratenko and E. Saldin, Part. Accel. 10, 207 (1980).

[15] Y. Derbenev, A. Kondratenko, and E. Saldin, Nucl. Instrum. Methods 193, 415 (1982).

[16] R. Bonifacio, C. Pellegrini, and L. Narducci, Opt. Commun. 50, 373 (1984).

[17] J. Murphy and C. Pellegrini, Nucl. Instrum. Methods Phys. Res., Sect. A 237, 159 (1985).

[18] W. Fawley, Phys. Rev. ST Accel. Beams 5, 070701 (2002).

[19] E. Saldin, E. Schneidmiller, and M. Yurkov, Opt. Commun. 148, 383 (1998).

[20] J. Goodman, Statistical Optics (Wiley, New York, 1985).

[21] E. Saldin, E. Schneidmiller, and M. Yurkov, Opt. Commun. 212, 377 (2002).

[22] H. Wiedemann, Synchrotron Radiation (Springer-Verlag, Berlin, 2003). 